\title{
Lower Locus Coeruleus MRI intensity in patients with late-life major depression
}

\author{
Andrés Guinea-Izquierdo Equal first author, 1, 2 , Mónica Giménez Equal frst author, 2 , Ignacio Martínez-Zalacaín ${ }^{1,2}$, Inés del Cerro ${ }^{1,2,3}$ \\ , Pol Canal-Noguer ${ }^{4,5,6}$, Gerard Blasco ${ }^{7}$, Jordi Gascón ${ }^{8}$, Ramon Reñé ${ }^{8}$, Inmaculada Rico ${ }^{8}$, Angels Camins ${ }^{7}$, Carlos \\ Aguilera $^{7}$, Mikel Urretavizcaya ${ }^{1,2,3}$, Isidre Ferrer ${ }^{9,10,11}$, José Manuel Menchón ${ }^{1,2,3}$, Virginia Soria ${ }^{\text {Corresp., 1, 2, }}{ }^{\text {, }}$, Carles \\ Soriano-Mas ${ }^{\text {Corresp. 2, 3,12 }}$ \\ 1 Department of Clinical Sciences / School of Medicine, University of Barcelona, Barcelona, Spain \\ 2 Department of Psychiatry / Bellvitge University Hospital, Bellvitge Biomedical Research Institute (IDIBELL), Hospitalet de Llobregat (Barcelona), Spain \\ 3 Network Center for Biomedical Research on Mental Health (CIBERSAM), Madrid, Spain \\ 4 B2SLab / Departament d'Enginyeria de Sistemes, Automàtica i Informàtica Industrial, Universitat Politècnica de Catalunya, Barcelona, Spain \\ 5 Networking Biomedical Research Centre in the subject area of Bioengineering, Biomaterials and Nanomedicine (CIBER-BBN), Madrid, Spain \\ 6 Institut de Recerca Pediàtrica, Hospital Sant Joan de Déu, Esplugues de Llobregat (Barcelona), Spain \\ 7 Imaging Diagnostic Institute (IDI), Bellvitge University Hospital, Hospitalet de Llobregat (Barcelona), Spain \\ 8 Dementia Diagnostic and Treatment Unit / Department of Neurology, Bellvitge University Hospital, Hospitalet de Llobregat (Barcelona), Spain \\ 9 Department of Pathology and Experimental Therapeutics / Institute of Neurosciences, University of Barcelona, Hospitalet de Llobregat (Barcelona), Spain \\ 10 Department of Pathologic Anatomy / Bellvitge University Hospital, Bellvitge Biomedical Research Institute-IDIBELL, Hospitalet de Llobregat (Barcelona), \\ Spain \\ 11 Network Center for Biomedical Research on Neurodegenerative diseases (CIBERNED), Madrid, Spain \\ 12 Department of Psychobiology and Methodology in Health Sciences, Universitat Autónoma de Barcelona, Bellaterra (Barcelona), Spain \\ Corresponding Authors: Virginia Soria, Carles Soriano-Mas \\ Email address: vsoria@bellvitgehospital.cat, csoriano@idibell.cat
}

Background. The locus coeruleus (LC) is the major noradrenergic source in the central nervous system. Structural alterations in the LC contribute to the pathophysiology of different neuropsychiatric disorders, which may increase to a variable extent the likelihood of developing neurodegenerative conditions. The characterization of such alterations may therefore help to predict progression to neurodegenerative disorders. Despite the LC cannot be visualized with conventional magnetic resonance imaging (MRI), specific MRI sequences have been developed to infer its structural integrity. Methods. We quantified LC signal Contrast Ratios (LCCRs) in late-life major depressive disorder (MDD) ( $n=37,9$ with comorbid $\mathrm{aMCl})$, amnestic Mild Cognitive Impairment $(\mathrm{aMCl})(\mathrm{n}=21$, without comorbid MDD), and healthy controls (HCs) ( $n=31)$, and also assessed the putative modulatory effects of comorbidities and other clinical variables. Results. LCCRs were lower in MDD compared to $\mathrm{aMCl}$ and $\mathrm{HCs}$. While no effects of aMCl comorbidity were observed, lower LCCRs were specifically observed in patients taking serotonin/norepinephrine reuptake inhibitors (SNRIs). Conclusion. Our results do not support the hypothesis that lower LCCRs characterize the different clinical groups that may eventually develop a neurodegenerative 
disorder. Conversely, our results were specifically observed in patients with late-life MDD taking SNRIs. Further research with larger samples is warranted to ascertain whether medication or particular clinical features of patients taking SNRIs are associated with changes in LC neurons. 


\section{"These authors contributed equally to the work}

\#Shared corresponding author

Andrés Guinea-Izquierdo, $\mathrm{MSc}^{1,2^{*}}$, Mónica Giménez, $\mathrm{PhD}^{1 *}$, Ignacio Martínez-Zalacaín, $\mathrm{MSc}^{1,2}$, Inés del Cerro, $\mathrm{MSc}^{1,2,3}$, Pol Canal-Noguer, $\mathrm{MSc}^{4,5,6}$, Gerard Blasco, $\mathrm{PhD}^{7}$, Jordi Gascón, $\mathrm{MD}^{8}$, Ramón Reñé, $\mathrm{MD}, \mathrm{PhD}^{8}$, Inmaculada Rico ${ }^{8}$, Àngels Camins $\mathrm{MD}^{7}$, Carlos Aguilera $\mathrm{MD} \mathrm{PhD}^{7}$, Mikel Urretavizcaya, $\mathrm{MD} \mathrm{PhD}^{1,2,3}$, Isidre Ferrer, $\mathrm{MD} \mathrm{PhD}^{9,10,11}$, José M Menchón, MD PhD ${ }^{1,2,3}$, Virginia Soria, $\mathrm{MD} \mathrm{PhD}{ }^{1,2,3 \#}$, Carles Soriano-Mas, $\mathrm{PhD}^{1,3,12 \#}$

1 Bellvitge Biomedical Research Institute-IDIBELL, Department of Psychiatry, Bellvitge University Hospital, L'Hospitalet de Llobregat, Barcelona, Spain

2 Department of Clinical Sciences, School of Medicine, University of Barcelona, Barcelona, Spain

3 Network Center for Biomedical Research on Mental Health (CIBERSAM), Carlos III Health Institute (ISCIII), Madrid, Spain

4 B2SLab, Departament d'Enginyeria de Sistemes, Automàtica i Informàtica Industrial, Universitat Politècnica de Catalunya, Barcelona, Spain

5 Networking Biomedical Research Centre in the subject area of Bioengineering, Biomaterials and Nanomedicine (CIBER-BBN), Madrid, Spain

6 Institut de Recerca Pediàtrica, Hospital Sant Joan de Déu, Esplugues de Llobregat, Spain

7 Imaging Diagnostic Institute (IDI), Bellvitge University Hospital, Hospitalet de Llobregat, Barcelona, Spain

8 Dementia Diagnostic and Treatment Unit, Department of Neurology, Bellvitge University Hospital, Hospitalet de Llobregat, Barcelona, Spain

9 Department of Pathology and Experimental Therapeutics, Institute of Neurosciences, University of Barcelona, Hospitalet de Llobregat, Barcelona, Spain

10 Bellvitge Biomedical Research Institute-IDIBELL, Department of Pathologic Anatomy, Bellvitge University Hospital, Hospitalet de Llobregat, Barcelona, Spain

11 Network Center for Biomedical Research on Neurodegenerative diseases (CIBERNED), Hospitalet de Llobregat, Barcelona, Spain

12 Department of Psychobiology and Methodology in Health Sciences, Universitat Autònoma de Barcelona, Bellaterra, Barcelona, Spain

\section{Corresponding Authors}


41

42 Carles Soriano-Mas

43

44 Department of Psychiatry, Bellvitge University Hospital, Bellvitge Biomedical Research

45 Institute-IDIBELL. Feixa Llarga s/n, 08907, L'Hospitalet de Llobregat, Barcelona, Spain

46 Email address: csoriano@idibell.cat

47

48 Virginia Soria

49

50 Department of Psychiatry, Bellvitge University Hospital, Bellvitge Biomedical Research

51 Institute-IDIBELL. Feixa Llarga s/n, 08907, L'Hospitalet de Llobregat, Barcelona, Spain.

52 Email address: vsoria @bellvitgehospital.cat 


\section{Abstract}

55

56

57

58

59

60

61

62

63

64

65

66

67

68

69

70

71

72 73

74

75

76

77

78

79

Background. The locus coeruleus (LC) is the major noradrenergic source in the central nervous system. Structural alterations in the LC contribute to the pathophysiology of different neuropsychiatric disorders, which may increase to a variable extent the likelihood of developing neurodegenerative conditions. The characterization of such alterations may therefore help to predict progression to neurodegenerative disorders. Despite the LC cannot be visualized with conventional magnetic resonance imaging (MRI), specific MRI sequences have been developed to infer its structural integrity.

Methods. We quantified LC signal Contrast Ratios (LCCRs) in late-life major depressive disorder (MDD) ( $n=37,9$ with comorbid aMCl), amnestic Mild Cognitive Impairment $(\mathrm{aMCl})(\mathrm{n}=21$, without comorbid MDD), and healthy controls $(\mathrm{HCs})(\mathrm{n}=31)$, and also assessed the putative modulatory effects of comorbidities and other clinical variables.

Results. LCCRs were lower in MDD compared to aMCl and HCs. While no effects of aMCl comorbidity were observed, lower LCCRs were specifically observed in patients taking serotonin/norepinephrine reuptake inhibitors (SNRIs).

Conclusion. Our results do not support the hypothesis that lower LCCRs characterize the different clinical groups that may eventually develop a neurodegenerative disorder. Conversely, our results were specifically observed in patients with late-life MDD taking SNRIs. Further research with larger samples is warranted to ascertain whether medication or particular clinical features of patients taking SNRIs are associated with changes in LC neurons. 


\section{Introduction}

82

83

84

85

86

87

88

89

90

91

92

93

94

95

96

97

98

99

100

101

102

103

104

105

106

107

108

109

110

111

112

113

114

115

116

117

118

119

120

The Locus Coeruleus (LC) is a small pontine nucleus with the largest group of noradrenergic (NA) neurons in the central nervous system (CNS) (Berridge \& Waterhouse, 2003; Dahlstroem $\&$ Fuxe, 1964). Its ascending and descending projections modulate neuronal activity in numerous targets throughout the CNS (Loughlin, Foote \& Fallon, 1982; Loughlin, Foote \& Grzanna, 1986; Loughlin, Foote \& Bloom, 1986; Samuels \& Szabadi, 2008). Different studies suggest that alterations in the LC, and, hence, in its projection network, may critically contribute to the onset as well as to the course and symptom profile of a range of neurological and psychiatric disorders (Betts et al., 2019; Charney, 1998; Delgado \& Moreno, 2000; Gannon et al., 2015; Leonard, 1997). Specifically, the LC has been suggested to be one of the initial sites of appearance of pathologically altered tau aggregates in preclinical stages of Alzheimer's disease (AD) (Braak et al., 2011; Braak and Del Tredici, 2015; Zarow et al., 2003), leading to compensatory increases in $\alpha 2 \mathrm{~A}$ adrenergic receptor levels in regions receiving noradrenergic input (Andrés-Benito et al., 2017). Moreover, there is evidence that loss of noradrenergic input exacerbates AD progression (Marien et al., 2004; Heneka et al., 2006; Grudzien et al., 2007).

One of the major limitations to investigate the role of the LC in brain disorders with in-vivo noninvasive techniques is that the $\mathrm{LC}$ is difficult to visualize with conventional magnetic resonance imaging (MRI) techniques. However, Sasaki et al. (2006) identified the LC in vivo as two bilateral hyperintensities adjacent to the floor of the fourth ventricle adapting the parameters of a traditional 2D T1-weighted fast spin echo sequence (for instance, using a higher number of averages and a high in-plane resolution with a large slice thickness). The molecular underpinnings of such hyperintense signal are still unknown (Betts et al., 2019), although it was originally thought to be related with the accumulation of neuromelanin in LC neurons.

Neuromelanin is a dark polymer pigment formed as a byproduct of catecholamine metabolism, and it can be found in the LC and substantia nigra pars compacta. The function of this macromolecule is not entirely known, but it has been shown that neuromelanin accumulates throughout life in noradrenergic LC neurons, binding to metals such as iron and copper (Enochs et al., 1989, 1997; Trujillo et al., 2017). Due to its ability to chelate metals, neuromelanin could be acting as a paramagnetic agent (Tosk et al., 1992), and perhaps neuromelanin could be a paramagnetic agent itself (Shima et al., 1997). These properties initially led some authors to hypothesize that neuromelanin could be causing the T1 shortening effects generating the LC contrast in fast spin echo T1 sequences (Sasaki et al., 2006).

Moreover, using MRI and post mortem histology, Keren et al. (2015) showed that T1-weighted LC hyperintensities co-localized with LC neurons with high neuromelanin concentration. Nevertheless, more recent research suggested that it is unlikely that LC hyperintensities are reflecting neuromelanin accumulation, but rather water content or general neuronal density

Peer) reviewing PDF | (2020:04:48357:3:0:NEW 23 Dec 2020) 
121 (Watanabe et al., 2019). In this sense, throughout the manuscript, we will avoid using the term

122

123

124

125

126

127

128

129

130

131

132

133

134

135

136

137

138

139

140

141

142

143

144

145

146

147

148

149

150

151

152

153

154

155

156

157

158 contrast ratios (LCCRs) seem to be a valid approximation to infer structural integrity of the LC (Betts et al., 2019).

There have been different reports of an overall lower LC signal in different neuropsychiatric conditions, such as major depressive disorder (MDD) (Sasaki et al., 2010; Shibata et al., 2008), schizophrenia (Shibata et al., 2008), amnestic mild cognitive impairment (aMCI) (Takahashi et al., 2015), and Alzheimer's or Parkinson's disease (Sasaki et al., 2006; Isaias et al., 2016; Matsuura et al., 2013; Miyoshi et al., 2013; Mukai et al., 2013; Ohtsuka et al., 2014), among others (for a review see Liu et al., 2017). It is not clear, however, to what extent such alterations show diagnostic specificity, which might be useful for clinical purposes (e.g., differential diagnosis, treatment selection, or prediction of clinical course). This could be important for disorders with partially overlapping clinical manifestations, such as late-life MDD and aMCI, two conditions typically appearing between the seventh and the eighth decades of life (Panza et al., 2010; Steffens, 2012).

People suffering from late-life MDD or aMCI have an increased risk for the later development of AD. Nevertheless, while aMCI is a common prodromal stage of $\mathrm{AD}$, with a progression rate to AD estimated between 6\% to 25\% per year (Petersen, 2001) (for a review see Hermida et al., 2012), late-life MDD is not that robustly associated with dementia onset, and results have been more heterogeneous. Overall, research suggests that people suffering from late-life MDD have an approximately twofold increased risk of developing AD (Byers \& Yaffe, 2011), although it is not clear whether depression is only a risk factor or may be considered a prodromal symptom of dementia (Byers \& Yaffe, 2011). Interestingly, moreover, co-occurrence of aMCI and depression seems to increase even more the probability to develop to AD, suggesting that both conditions have an additive or synergic effect (Modrego \& Ferrandez, 2004; Gabryelewicz et al., 2007). In this context, it is not clear whether LC MRI intensity discriminates between these disorders and their comorbidity, and, therefore, to what extent this measurement could contribute to identify subjects at a higher risk of developing a neurodegenerative disorder.

In this study, we aimed at comparing LC MRI intensity between patients with late-life MDD and individuals with aMCI, which, for reference purposes, were also compared to a group of healthy controls (HCs). Moreover, as a secondary aim, we specifically assessed a group of individuals presenting comorbidity between the two diagnoses, and evaluated the modulatory effect on our findings of medication and other clinical variables. According to previous literature, we hypothesized that LC MRI intensity will discriminate between the clinical groups and HCs, and also across patients with late-life MDD, aMCI and a comorbid presentation of both disorders.

Peer) reviewing PDF | (2020:04:48357:3:0:NEW 23 Dec 2020) 


\section{Materials \& Methods}

160

161

162

163

164

165

166

167

168

169

170

171

172

173

174

175

176

177

178

179

180

181

182

183

184

185

186

187

188

189

190

191

192

193

194

195

196

197

198

\section{Participants}

The sample consisted of 89 individuals between 60 and 76 years of age. These were divided into three groups: 37 patients with a primary diagnosis of late-life MDD (11 males, mean age [standard deviation, SD]: 68 [4.1] years), 21 subjects with aMCI (9 males, mean age: 71.5 [2.7] years), and $31 \mathrm{HCs}$ from the same geographical area (11 males, mean age [SD]: 67.7 [4.1] years. Subjects from the clinical groups were consecutively recruited from the Psychiatry and Neurology Departments of Bellvitge University Hospital (Barcelona, Spain), and HCs through advertisements and word-of-mouth. All participants were interviewed with the MiniInternational Neuropsychiatric Interview (MINI) (Sheehan et al., 1998). MDD diagnoses were established by two experienced psychiatrists (VS and MU) according to DSM-IV-TR criteria, and disorder severity was estimated with the Hamilton Depression Rating Scale (HDRS) (Hamilton, 1960) and the Geriatric Depression Scale (GDS) (Sheikh \& Yesavage, 1986; Yesavage et al., 1982), which were, however, not used for diagnostic purposes. Amnestic MCI diagnoses were based on a syndromic categorical cognitive staging approach rather than on the use of biomarkers (Jack et al., 2018). Diagnosis was established by consensus of two experienced neurologists (JG and RR) and one neuropsychologist (IR) following Petersen criteria (Petersen, 2004), including: 1) complaints of memory loss confirmed by informants, 2) objective long-term memory impairments (scores $1.5 \mathrm{SD}$ below mean age and education adjusted normative values in the delayed recall test from the Wechsler Memory Scale III (WMS-III) (Wechsler, 2004) and the brief neuropsychological battery NBACE (Alegret et al., 2012), 3) preserved general cognitive functioning (i.e., scores higher than 23 for literate people and higher than 18 for illiterates in the Spanish version of the Mini Mental State Examination (MMSE) (Folstein \& Folstein, 1975), and 4) intact or mildly impaired daily living abilities (Clinical Dementia Rating (CDR) (Morris,1993) scores of 0.5). All participants were also administered the Vocabulary subtest of the Wechsler Adult Intelligence Scale, Third Edition (WAIS-III) (Wechsler, 1999), to estimate the premorbid intelligence quotient (IQ) (García-Lorenzo et al., 2013). Importantly, aMCI participants did not present past or current comorbidity with MDD, while nine out of the 37 patients with MDD presented a comorbid aMCI diagnosis. In all cases, medication was not changed and was kept at stable doses for at least one month before MRI acquisition. Fourteen out of $21 \mathrm{aMCI}$ subjects were taking antidepressants for conditions other than MDD, such as impaired psychosocial adjustment, insomnia or headache. Table 1 summarizes the sociodemographic and clinical characteristics of the study sample, while information about antidepressant medication is provided in Table 2.

Exclusion criteria for the study participants included: 1) ages $<60$ or $>76$ years, 2) past or current diagnosis of other major psychiatric disorders including substance abuse or dependence (except nicotine), 3) intellectual disability/neurodevelopmental disorders, 4) neurological disorders, 5)

Peer) reviewing PDF | (2020:04:48357:3:0:NEW 23 Dec 2020) 
199

200

201

202

203

204

205

206

207

208

209

210

211

212

213

214

215

216

217

218

219

220

221

222

223

224

225

226

227

228

229

230

231

232

233

234

235

236

237

238

239

Hachinski Ischemic Score $>5$ to exclude individuals with a high probability of vascular-derived cognitive deficits, 6) presence of dementia according to the DSM-IV-TR criteria and/or a CDR score $>1,7$ ) severe medical conditions, 8) electroconvulsive therapy in the previous year, 9) conditions preventing neuropsychological assessment or MRI procedures (e.g., blindness, deafness, claustrophobia, pacemakers or cochlear implants), and 10) gross abnormalities in the MRI scan. We also evaluated the presence of vascular pathology in our brain region of interest (pons), finding no significant alterations preventing accurate image processing and analysis.

The study was approved by The Clinical Research Ethics Committee (CEIC) of Bellvitge University Hospital (reference PR156/15, February 17th 2016) and performed in accordance with the ethical standards laid down in the 1964 Declaration of Helsinki and its later amendments (revised in 2013). All participants gave written informed consent to participate in the study.

\section{MRI scanning protocol}

All scans were performed in a 3T Philips Ingenia Scanner (Koninklijke Philips N.V., Netherlands), using a 32-channel head coil. Following previous reports (Sasaki et al., 2006), we obtained a modified T1-weighted fast spin-echo sequence of the brainstem for LC visualization (see examples of individual participants in Supplementary Figure S1). Acquisition parameters were: TR $600 \mathrm{~ms}$, TE $15 \mathrm{~ms}$, 15 slices; $2.5 \mathrm{~mm}$ slice thickness, $0 \mathrm{~mm}$ gap, matrix size 404x250, FOV $170 \times 170 \mathrm{~mm}^{2}$, acquisition voxel size $0.42 \times 0.68 \times 2.5 \mathrm{~mm}^{3}$, reconstructed voxel size $0.39 \times 0.39 \times 2.5 \mathrm{~mm}^{3}, \mathrm{FA} 90^{\circ}, 6 \mathrm{NEX}$ (online averaging), and a total scan time of 15 minutes. The sections were acquired in the oblique axial direction perpendicular to the floor of the fourth ventricle, covering from the posterior commissure to the inferior border of the pons. Axial T1weighted turbo-gradient-echo high-resolution whole-brain anatomical images (233 slices, $\mathrm{TR}=10.46 \mathrm{~ms}, \mathrm{TE}=4.79 \mathrm{~ms}$, flip angle $=8^{\circ}, \mathrm{FOV}=240 \times 240,0.75 \times 0.75 \mathrm{~mm}$ isotropic voxels) were additionally obtained for pre-processing purposes and to discard gross structural pathology. Finally, we also obtained a 2D FLAIR sequence in the axial plane ( 38 slices, $\mathrm{TR}=10000 \mathrm{~ms}$, $\mathrm{TE}=140 \mathrm{~ms}, \mathrm{TI}=2700 \mathrm{~ms}, \mathrm{FOV}=230 \times 186,0.8 \times 1 \times 3 \mathrm{~mm}$ voxels, $0.6 \mathrm{~mm}$ gap) to assess vascular pathology and other potential radiological abnormalities. Throughout the acquisition protocol, we used foam pads and made sure that patients' head was comfortably placed within the head coil to avoid excessive movement.

\section{Localization and quantification of the $\mathrm{LC}$}

To quantify LC MRI intensity, we used a semi-automated "in-house" algorithm. We focused our analysis on the region of the dorsal pons. Specifically, our approach was based on previous studies (García-Lorenzo et al, 2013) and consisted of two main steps: 
240 A): Delineation of regions of interest (ROIs): We defined four rectangular ROIs onto the

241 Montreal Neurological Institute 152 [MNI 152] template ( $0.5 \mathrm{~mm}$ isotropic resolution) using the

242 Statistical Parametric Mapping (SPM 12) software (http://www.fil.ion.ucl.ac.uk/spm/).

243 Specifically, two of these ROIs were symmetrically located in the left and right areas of the

244 dorsal pons where the LC is expected to be found, adjacent to the floor of the fourth ventricle

245 and extending to the level of the inferior colliculi. Importantly, we explicitly avoided including

246 in these ROIs other hyperintense regions, such as the substantia nigra, to ensure that the LC was

247 the highest signal intensity. The other two ROIs covered the mid portions of the adjacent left and

248 right pontine tegmentum (again, avoiding the inclusion of the substantia nigra), and were used as

249 a reference to standardize LC intensity values (see below). These ROIs, drawn in normalized

250 MNI space, were then denormalized to the native space of each participant. However, due to the

251 small size of the locus coeruleus and the anisotropic voxel size of the images, the LC sensitive

252 sequence was not manipulated and was used as the target image, therefore avoiding interpolation

253 artifacts and preserving the original signal. Consequently, we first linearly coregistered, for each

254 participant and in native space, the whole-brain T1 image to the LC sensitive image. Next, the

255 tissue probability map (TPM) and the four ROIs in MNI space were coregistered (linear

256 coregistration) to the whole-brain T1 image from the previous step, and such individual whole-

257 brain T1 images were non-linearly normalized to the coregistered TPM, which generated a

258 deformation field. The inverse of the deformation field was calculated by using the deformation

259 function in SPM, taking the LC sensitive image as the image to base the inversion on. The four

260 ROIs were then non-linearly denormalized to each participant native space by applying the

261 inverse of the deformation fields with a $4^{\text {th }}$ degree b-spline interpolation. Finally, these ROIs

262 were binarized using a trilinear interpolation and a cut-off value of 0.1 (using the ImCalc

263 function; i1 $>0.1$ ) in order to better delimitate the ROI area to be extracted; then, each binarized

264 ROI was applied to the LC-sensitive image (ImCalc function; i1.*i2), which effectively removed

265 from these images all the information outside the ROIs. Therefore, the final output of the process

266 were 4 images for participant, in native space, corresponding to the projection of each ROI into

267 the LC-sensitive image. This approach is summarized in Supplementary Figure S2. Likewise,

268 Supplementary Figure S3 displays the accuracy of the coregistration between the LC, the

269 reference ROI and the brainstem structures in native space.

270

271 B): Quantification of the LC signal with a growing algorithm. This second step was implemented 272 using an in-house growing algorithm, programmed in MATLAB version 9.3 (R2017b) (The

273 MathWorks Inc, Natick, Massachusetts), which was applied to the final output images from step

274 A) that encompassed the LC region. Specifically, following García-Lorenzo et al. (2013), who

275 defined LC as "the area of 10-connected voxels with the brightest intensity", the current

276 algorithm searched throughout these images the 10 clusters of 10 contiguous voxels (face and/or

277 edge and/or vertex contiguity) with the highest intensity (highest mean intensity of the cluster)

278 (see Figure 1). These clusters were visually inspected for their anatomical correspondence with

279 the region of the LC. In case the cluster with highest intensity was deemed to be outside the LC 
280 region, the second cluster was inspected, and so on. Finally, we obtained, for each participant, 281 the 10-voxels cluster of highest signal intensity within the LC region, as well as the mean 282 intensity value and peak coordinate for this cluster $\left(\mathrm{S}_{\mathrm{LC}}\right)$. In agreement with our interest in 283 assessing the intensity of LC, this 10-voxels average represents a good estimation of mean LC 284 intensity and its presumed neural integrity (Liu et al., 2017). This is different from a volume 285 measure of the nucleus; also, we did not discriminate intensities across the different territories of 286 the LC (García-Lorenzo et al., 2013).

287

288 The mean signal intensity from the reference pontine tegmentum cluster was also obtained for 289 each participant $\left(\mathrm{S}_{\mathrm{REF}}\right)$. This value was used to control for putative differences in global signal 290 intensity across participants. We used the following formula to calculate the LC contrast ratio 291 (CR) (Liu et al., 2017): $\mathrm{CR}=\left(\mathrm{S}_{\mathrm{LC}}-\mathrm{S}_{\mathrm{REF}}\right) / \mathrm{S}_{\mathrm{REF}}$. This $\mathrm{CR}$ was calculated for each participant and 292 for both LC (left and right), obtaining two different CRs for each participant (CR Left and CR

\section{Statistical analysis}

299

300

Differences in demographical and clinical features were contrasted across the three study groups

301

302

303

304

305

306

307

308

309

310

311

312

313

314

315

316

317

318 with the Kruskal-Wallis test for continuous variables and the Chi-Square test for categorical variables. Differences in average LCCR were also assessed across the study groups using the Kruskal-Wallis test. Having in mind that LC MRI contrast ratios interact with age (Liu et al., 2019) and that structural and functional differences between men and women have been found in this region (Bangasser et al, 2016; Mulvey et al, 2018), average LCCR comparisons were controlled for age and sex. For this, we created a new variable (standardized residual of average LCCR, sraLCCR) extracting the effect of both variables, as non-parametrical tests do not allow nuisance covariates. When the Kruskal-Wallis test showed significant differences across the three groups, pairwise comparisons were assessed using the Mann-Whitney's U test. Moreover, we used Spearman's Rho $\left(r_{\mathrm{s}}\right)$ correlations to evaluate the possible associations between sraLCCR and different clinical features (i.e., dose and duration of treatments, MMSE).

Finally, we analyzed the modulatory effect of treatment (e.g., treatment type, duration or dose) on sraLCCR with the Kruskal-Wallis test and Spearman correlations (for categorical and continuous variables, respectively). When adequate, statistical significance was adjusted with a Bonferroni correction for multiple testing. We also calculated Cliff's $\delta$ statistic (Cliff, 1996) to estimate effect sizes. 
320

321

322

323

324

325

326

327

328

329

330

331

332

333

334

335

336

337

338

339

340

341

342

343

344

345

346

347

348

349

350

351

352

353

354

355

356

357

358

359

\section{Results}

Data on sociodemographic and clinical characteristics of study participants are displayed in Table 1. Due to the consecutive recruitment strategy, the groups differed in age $(H=13.38$, $\mathrm{p}=0.001$, degrees of freedom $(\mathrm{df})=2$ ), with the aMCI group showing the highest mean age, while the MDD group did not differ from HCs. Also, we found a significant negative correlation between the right LCCR and age $\left(r_{s}=-0.218, p=0.04\right)$. In any case, as described above, all analyses were controlled for age and sex.

The Kruskal-Wallis test showed significant across-group (MDD n=37, aMCI n=21, HCs n=31) differences in $\operatorname{sraLCCR}(\mathrm{H}=16.64, \mathrm{df}=2, \mathrm{p}=0.001)$. In post-hoc analyses, it was observed that MDD subjects displayed a lower $\operatorname{sraLCCR}$ in comparison to aMCI $(\mathrm{U}=193, \mathrm{Z}=-3.16, \mathrm{p}<0.005, \delta$ $($ Cliff's delta effect size $)=-0.53)$ and HCs $(U=281, Z=-3.60, p<0.001, \delta=-0.51)$. See Table 3 and Figure 2a. Importantly, our groups did not differ in the signal intensity from the reference region $(\mathrm{H}=3.47, \mathrm{df}=2, \mathrm{p}=0.177)$, and, therefore, $\operatorname{sraLCCR}$ differences were attributable to differences in LC signal intensity.

We did not observe any significant effects of aMCI comorbidity in patients with MDD, since no significant differences were found in sraLCCR between the subgroup of MDD without aMCI $(n=28)$ and MDD patients with comorbid aMCI $(n=9)(U=95, Z=-1.10, p=0.272, \delta=-0.25)$. Likewise, both groups significantly differed from HCs and the aMCI group (see Supplementary Figure S4). Moreover, as depicted in Supplementary Figure S5, we observed no significant correlations between sraLCCR values and MMSE scores for any of the study groups.

When evaluating the possible effects of medication (see Figure $2 b$ ), we observed that patients with MDD taking Serotonin and Norepinephrine Reuptake Inhibitors (SNRIs) $(n=25)$ had a significantly lower sraLCCR compared to HCs $(\mathrm{n}=31)(\mathrm{U}=143, \mathrm{Z}=-4.03, \mathrm{p}<0.001, \delta=-0.63)$ and to aMCI patients $(n=21)(U=94, Z=-3.72, p<0.001, \delta=-0.64)$. However, we did not observe significant differences in sraLCCR between MDD patients taking $(\mathrm{n}=25)$ and not taking $(\mathrm{n}=12)$ SNRIs, although sraLCCR of MDDs patients not taking SNRIs did not differ from $\mathrm{HCs}(\mathrm{n}=31)$ $(\mathrm{U}=138, \mathrm{Z}=-1.30, \mathrm{p}=0.194, \delta=-0.26)$ or aMCI patients $(\mathrm{n}=21)(\mathrm{U}=99, \mathrm{Z}=-1.01, \mathrm{p}=0.312$, $\delta=-0.21)$. Although it was not possible to properly evaluate the effect of SNRIs due to the small sample size of the group of MDD subjects exclusively taking SNRIs $(n=5)$, comparing the group of MDD patients taking SNRIs alone with the rest of MDD patients $(n=32)$ and with patients taking SNRIs and other psychiatric medications $(n=20)$ we did not find significant differences $(\mathrm{U}=60, \mathrm{Z}=-0.89, \mathrm{p}=0.374, \delta=-0.25$; and $\mathrm{U}=43, \mathrm{Z}=-0.48, \mathrm{p}=0.634, \delta=-0.14)$. Importantly, we did not find any significant differences between MDD patients taking and not taking SNRIs in clinical variables such as severity, disease duration or treatment resistance. These results are presented in Supplementary Table S1. 
360 Importantly, we repeated the analysis by subdividing the MDD group into those who took ( $\mathrm{n}=31)$ 361 and those who did not take $(\mathrm{n}=6)$ medications with noradrenergic effect (SNRIs + Tricyclics +

362 Mirtazapine + Vortioxetine + Agomelatine + Bupropion), obtaining similar results: MDD taking 363 noradrenergic medication had a significant lower sraLCCR compared to HCs $(n=31)(U=209$, $364 \mathrm{Z}=-3.82, \mathrm{p}<0.001, \delta=-0.57)$ and the aMCI group $(\mathrm{n}=21)(\mathrm{U}=143, \mathrm{Z}=-3.40, \mathrm{p}<0.005, \delta=-0.56)$. 365 Again, although we did not observe significant differences in sraLCCR between MDD patients 366 taking and not taking noradrenergic medications, sraLCCR of MDD patients not taking

368

369

370

371

372

373

374

375

376

377

378

379

380

381

382

383

384

385

386

387

388 noradrenergic medications did not differ from $\mathrm{HCs}(\mathrm{n}=31)(\mathrm{U}=72, \mathrm{Z}=-0.86, \mathrm{p}=0.387, \delta=-0.23)$ or aMCI patients $(\mathrm{n}=21)(\mathrm{U}=50, \mathrm{Z}=-0.76, \mathrm{p}=0.448, \delta=-0.21)$. Also, MDD patients exclusively taking noradrenergic medications other than SNRIs $(n=6)$ did not differ from patients taking SNRIs ( $n=25)$, not taking noradrenergic medications $(n=6)$, or the HCs and aMCI groups (see Supplementary Table S2). Finally, we also assessed whether subjects taking adrenergic medication for non-mental health purposes (i.e., beta-blockers; 4 HCs, 1 aMCI and 7 MDD) showed differences in sraLCCRs, finding non-significant effects.

Unfortunately, it was not possible to properly evaluate the effect of Selective Serotonin Reuptake Inhibitors (SSRIs) due to the small sample size of the group of MDD subjects exclusively taking SSRIs ( $n=3$ ). Nevertheless, comparing the group of MDD patients taking SSRIs alone or in combination with other medications $(n=6)$ with HCs and patients with aMCI, we did not find significant differences after Bonferroni correction $(U=32, Z=-2.51, p=0.012, \delta=-0.66$, and $U=20$, $\mathrm{Z}=-2.51, \mathrm{p}=0.012, \delta=-0.68$; Bonferroni $\mathrm{p}$ thresholded at $\mathrm{p}=0.0083$ ).

Finally, we did not find significant correlations between dose or duration of any of the treatments and $\operatorname{sraLCCR}$.

Unless otherwise indicated, all the above significant findings survived Bonferroni correction for multiple comparisons. 
390

391

392

393

394

395

396

397

398

399

400

401

402

403

404

405

406

407

408

409

410

411

412

413

414

415

416

417

418

419

420

421

422

423

424

425

426

427

428

429

\section{Discussion}

Our study compared LC MRI signal intensity between two groups of subjects with brain disorders conferring an increased risk for dementia. We observed that averageLCCR was significantly lower in late-life MDD patients compared to aMCI and HC groups, but no evidence of additive effects between depression and aMCI. Moreover, such lower LC intensity was associated with the medication used by participants, since significant effects were indeed only observed in the subgroup of MDD patients taking SNRIs.

Our results are in overall agreement with the monoamine hypothesis of depression (Delgado, 2000), which suggests that MDD patients show significant alterations in monoaminergic neurotransmission, and, more specifically, with previous neuroimaging studies reporting a lower LC intensity in patients with MDD compared to HCs (Sasaki et al., 2010; Shibata et al., 2008). Previous reports have shown that NA plays a major role in the pathophysiology of depression (Delgado \& Moreno, 2000; Moret, 2011), with the LC projecting to emotion- and cognitionrelated areas implicated in the pathophysiology of depression (Stahl, 2008).

In any case, we also observed that this lower LC intensity was specific of MDD patients taking dual action antidepressants (SNRIs). There could be two different explanations for this. On the one hand, if we consider that LC MRI contrast is directly or indirectly associated to the accumulation of neuromelanin compounds (Betts et al., 2019), we could speculate about the nature of such accumulation and the molecular effect of dual antidepressant treatment. Neuromelanin is an autophagic byproduct resulting from the metabolism of NA and other catecholamines (German et al., 1988; Graham, 1979), and its accumulation increases with age in different brain nuclei, such as the substantia nigra and the LC (Clewett et al., 2016). Since SNRIs antidepressants inhibit NA reuptake, the levels of this neurotransmitter are increased at the synaptic cleft, what could lead to a decrease in intracellular NA synthesis and metabolism. Sustained over time, this decrease in NA synthesis and degradation could result in a decreased neuromelanin accumulation in the LC. However, this could have been a strong argument if significant correlations between LC signal intensity and SNRIs treatment dose and duration had been observed, but this was not the case in our study. Moreover, there are no evidences of the existence of an enzymatic mechanism capable of inducing degradation or removing neuromelanin (Fedorow et al., 2006; Halliday et al., 2006), and, therefore, it is not possible to argue that noradrenergic antidepressants may be promoting neuromelanin degradation in LC neurons.

On the other hand, the possibility exists that particular clinical features of the subgroup of MDD subjects who are taking SNRIs might be associated with the lower LCCR in this group of patients. In our sample, MDD patients taking SNRIs did previously fail to respond to treatments with SSRIs. Speculatively, this treatment failure could be related to an impaired NA function 
430 reflected in lower MRI LCCR that required the active modulation of NA neurotransmission.

431 Although treatment with dual action antidepressant does not seem to normalize LCCR intensity

432 levels, prospective research on the interaction between antidepressant medication with

433 noradrenergic action and LC signal intensity, carefully controlling for doses and treatment

434 duration, is warranted to elucidate this issue.

435

436

437

Contrary to our initial expectations, we did not observe any significant effect of aMCI on LC

438 signal intensities, which prevents suggesting LC MRI intensity as a putative risk imaging marker

439

440

441

442

443

444

445

446

447

448

449

450

451

452

453

454

455

456

457

458

459

460

461

462

463

464

465

466

467

468

469 for $\mathrm{AD}$. These results contradict previous research showing not only an early loss of LC neurons in AD (Szot et al, 2006), but also lower LC intensity values in aMCI versus HCs (Takahashi et al, 2015). Critically, however, that study did not exclude patients with comorbid depression, while our group of aMCI individuals did not show comorbidity with major depression. Therefore, we propose that MDD symptoms may be actually more relevantly accounting for lower LC signal intensity. In agreement with this, we have also observed that the presence of comorbid aMCI in patients with MDD did not significantly contribute to further lowering LC signal intensities.

This study is not without limitations. First, since we did not obtain data on cerebrospinal fluid or PET imaging biomarkers, we used a syndromic categorical cognitive staging approach and we cannot confirm that our patients were indeed from the AD continuum and will ultimately develop AD. Second, longitudinal studies with larger and carefully characterized samples are highly encouraged in order to better explore the interactions over time of group findings with clinical and sociodemographic variables. This will probably allow identifying clinically relevant subgroups of individuals as a function of their LC signal intensities and contribute to better estimate the likelihood of a degenerative course. Third, we are not using a quantitative MRI method, although other MRI sequences, such as Magnetization Transfer (MT), may provide quantitative indices (Ramani et al., 2002; Sled and Pike, 2001) that have been used for assessing LC integrity (Trujillo et al., 2019). As many other groups, we used a Spin Echo (SE) sequence for assessing LC neuronal integrity (Liu et al., 2017), although MT based sequences seem to be a better option to perform absolute signal quantifications (Trujillo et al., 2019) and to more precisely localize the LC with isotropic resolution and increased contrast-to-noise ratio, especially at higher-field strengths (Priovoulos et al., 2018). We performed a relative quantification taking another brainstem region as the reference area to control for intensity fluctuations across participants, and, importantly, groups did not significantly differ in the intensity of this reference region, indicating that our group differences stem from differences in LC intensity. Nevertheless, further studies combining quantitative and non-quantitative approaches are probably needed to perform better estimations of neural integrity in the LC (Betts et al., 2019). Likewise, the present approach did not allow quantifying the volume of the LC, which would have required performing a manual segmentation of the nucleus, and, therefore, we can only infer changes in LC integrity from changes in MRI signal intensity. If such signal 
470 intensity is related to neuromelanin accumulation, LC integrity will be probably

471 reflectingpreserved adrenergic activity. By contrast, if such signal is related to higher neuronal

472 density or water content, as recently suggested (Watanabe et al., 2019), LC integrity will be

473 reflecting a lack of regional atrophy and/or a good osmotic balance in the context of a normal

474 neuronal metabolism.

475

476

477

In the context of limitations, it is also important to note that there are studies suggesting that age-

478 related variance should be considered when exploring structural abnormalities in the LC (Shibata et al, 2006; Liu et al., 2019). Moreover, these age effects may probably show a non-homogenous

479 distribution along the rostral-caudal axis of the nucleus (Betts et al, 2017; Dahl et al., 2019; Liu

480 et al., 2019). Thus, age-related declines in LCCR have been mainly observed in the rostral part of the LC in healthy subjects (Liu et al., 2019), being such decline in rostral LCCRs related to

482

483

484

485

486

487

488

489

490

491

492

493

494

495

496

497

498

499

500

501

502

503

504

505

506

507

508

509

poorer memory performance in neuropsychological testing (Dahl et al., 2019). Although we controlled for age and sex, our algorithm did not allow distinguishing between the rostral and the caudal parts of the nucleus, which may have partially confounded our findings. Moreover, white matter from the pontine tegmentum, used here as reference, has also been shown to increase its T1 intensity with age, probably as a consequence of the highly protracted cycle of myelination of this region, extending up to 70 or 80 years of age (Yakolev and Lecours, 1967). Despite our statistical control for age, and although our groups did not differ in the signal intensity of this reference region, this may have also partially confounded our findings.

\section{Conclusions}

To sum up, LC signal intensity does not seem to allow identifying subjects with cognitive profiles related to prodromal phases of AD. Conversely, it seems to be associated to late-life MDD. Further research with larger samples should ascertain whether medication aimed at modulating noradrenergic neurotransmission may be playing a role in these findings or, conversely, changes in LC neurons are associated with a particular clinical profile preferentially observed in patients responding to noradrenergic medication. It also remains to be determined if these findings may contribute to optimizing treatment of patients with late-life MDD.

\section{Acknowledgements}

The authors are grateful to all the study participants and their families, and to the staff and technicians of Bellvitge University Hospital and Duran i Reynals Hospital who helped to recruit the sample for this study. We thank CERCA Programme/Generalitat de Catalunya for institutional support.

Peer) reviewing PDF | (2020:04:48357:3:0:NEW 23 Dec 2020) 
510

\section{1}

512

513 Conceptualization, I.F., J.M.M., V.S. and C.S-M.; Methodology, A.G-I., I.M-Z., I.dC., G.B. and 514 C.S-M.; Software, I.M-Z. and P.C.; Validation, A.G-I., I.M-Z. and C.S-M.; Formal Analysis, 515 A.G-I., M.G. and I.M-Z.; Investigation, A.G-I., I.dC., J.G., R.R., I.R., A.C., M.U. and V.S.; 516 Resources, C.A., I.F., J.M.M. and C.S-M.; Data Curation, J.G., R.R., I.R., A.C., C.A., M.U., 517 V.S., J.M.M. and C.S-M.; Writing - Original Draft Preparation, A.G-I., M.G. and C.S-M.; 518 Writing - Review \& Editing, All authors; Visualization, A.G-I., M.G., I.dC. and C.S-M.;

519 Supervision, C.S-M; Project Administration, V.S. and C.S-M.; Funding Acquisition, I.F., J.M.M. 520 and C.S-M.

521

522

\section{Conflicts of Interest}

524

525

526

527

528

529

530

531

532

533

534

535

536

537 The rest of authors have nothing to disclose nor have any financial relationships with commercial 538 interests. 


\section{References}

541

542 Alegret, M.; Espinosa, A.; Vinyes-Junqué, G.; Valero, S.; Hernández, I.; Tárraga, L.; Becker, 543 J.T.; Boada, M. Normative data of a brief neuropsychological battery for Spanish individuals 544 older than 49. J Clin Exp Neuropsychol 2012, 34, 209-219.

545 Andrés-Benito, P.; Fernández-Dueñas, V.; Carmona, M.; Escobar, L.A.; Torrejón-Escribano, B.; 546 Aso, E; Ciruela, F; Ferrer I. Locus coeruleus at asymptomatic early and middle Braak stages of 547 neurofibrillary tangle pathology. Neuropathol Appl Neurobiol 2017, 43, 373-392.

548

549

Bangasser, D. A., Wiersielis, K. R., \& Khantsis, S. Sex Differences in the Locus Coeruleus-

550

551 Norepinephrine System and its Regulation by Stress. Brain Research 2016, 1641 (PtB), 177188.

552

553

Berridge, C.W.; Waterhouse, B.D. The locus coeruleus-noradrenergic system: modulation of

554 behavioral state and state-dependent cognitive processes. Brain Res. Brain Res. Rev. 2003, 42,

555 33-84.

556

557

558

Betts, M.J.; Cardenas-Blanco, A.; Kanowski, M.; Jessen, F.; Düzel, E. In vivo MRI assessment

559

560 of the human locus coeruleus along its rostrocaudal extent in young and older adults.

Neuroimage 2017, 163, 150-159.

561

Betts, M.J.; Kirilina, E.; Otaduy, M.C.G.; Ivanov, D.; Acosta-Cabronero, J.; Callaghan, M.F.;

562 Lambert, C.; Cardenas-Blanco, A.; Pine, K.; Passamonti, L.; Loane, C.; Keuken, M.C.;

563 Trujillo, P.; Lüsebrink, F.; Mattern, H.; Liu, K.Y.; Priovoulos, N.; Fliessbach, K.; Dahl, M.J.;

563 Maaß, A.; Madelung, C.F.; Meder, D.; Ehrenberg, A.J.; Speck, O.; Weiskopf, N.; Dolan, R.; Inglis, B.; Tosun, D.; Morawski, M.; Zucca, F.A.; Siebner, H.R.; Mather, M.; Uludag, K.;

565 Heinsen, H.; Poser, B.A.; Howard, R.; Zecca, L.; Rowe, J.B.; Grinberg, L.T.; Jacobs, H.I.L.; Düzel, E.; Hämmerer, D. Locus coeruleus imaging as a biomarker for noradrenergic dysfunction in neurodegenerative diseases. Brain 2019, 142, 2558-2571.

568

569

Braak, H.; Del Tredici, K. The preclinical phase of the pathological process underlying sporadic

570 Alzheimer's disease. Brain 2015, 138, 2814-2833.

571

572

Braak, H.; Thal, D.R.; Ghebremedhin, E.; Del Tredici, K. Stages of the pathologic process in 573 alzheimer disease: Age categories from 1 to 100 years. J Neuropathol Exp Neurol 2011, 70, 960574969.

575

576 Byers, A.L.; Yaffe, K. Depression and risk of developing dementia. Nat Rev Neurol 2011, 7, 577 323-331.

578 Charney, D.S. Monoamine dysfunction and the pathophysiology and treatment of depression. $J$ 579 Clin Psychiatry 1998, 59 Suppl 14, 11-14. 
580 Clewett, D.V.; Lee, T.-H.; Greening, S.; Ponzio, A.; Margalit, E.; Mather, M. Neuromelanin 581 marks the spot: identifying a locus coeruleus biomarker of cognitive reserve in healthy aging. 582 Neurobiol. Aging 2016, 37, 117-126.

583 Cliff, N. Answering Ordinal Questions with Ordinal Data Using Ordinal Statistics. Multivariate 584 Behav Res 1996, 31, 331-350.

585 Dahl, M.J.; Mather, M.; Düzel, S.; Bodammer, N.C.; Lindenbergr, U.; Kühn, S.; Werkle586 Bergner, M. Rostral locus Coeruleus integrity is associated with better memory performance in

587

588

589

590

591

592

593

594

595

596

597

598

599

600

601

602

603

604

605

606

607

608

609

610

611

612

613

614 older adults. Nature Human Behaviour 2019, 3, 1203-1214.

Dahlstroem, A.; Fuxe, K. Evidence for the existence of monoamine-containing neurons in the central nervous system. I. Demonstration of monoamines in the cell bodies of brain stem neurons. Acta Physiol Scand Suppl 1964, Suppl 232, 1-55.

Delgado, P.L.; Moreno, F.A. Role of norepinephrine in depression. J Clin Psychiatry 2000, 61 Suppl 1, 5-12.

Delgado, P.L. Depression: the case for a monoamine deficiency. J Clin Psychiatry 2000, 61 Suppl 6, 7-11.

Enochs, W.S.; Hyslop, W.B.; Bennett, H.F.; Brown, R.D.; Koenig, S.H.; Swartz, H.M. Sources of the increased longitudinal relaxation rates observed in melanotic melanoma. An in vitro study of synthetic melanins. Investigative Radiology 1989, 24, 794-804.

Enochs, W.S.; Petherick, P.; Bogdanova, A.; Mohr, U.; Weissleder, R. Paramagnetic metal scavenging by melanin: MR imaging. Radiology 1997, 204, 417-423.

Fedorow, H.; Halliday, G.M.; Rickert, C.H.; Gerlach, M.; Riederer, P.; Double, K.L. Evidence for specific phases in the development of human neuromelanin. Neurobiol. Aging 2006, 27, 506512.

Folstein, M.F.; Folstein, S.E.; McHugh, P.R. "Mini-mental state”. A practical method for grading the cognitive state of patients for the clinician. J Psychiatr Res 1975, 12, 189-198.

Gabryelewicz, T.; Styczynska, M.; Luczywek, E.; Barczak, A.; Pfeffer, A.; Androsiuk, W.; Chodakowska-Zebrowska, M.; Wasiak, B.; Peplonska, B.; Barcikowska, M. The rate of conversion of mild cognitive impairment to dementia: predictive role of depression. Int J Geriatr Psychiatry. 2007;22:563-567.

Gannon, M.; Che, P.; Chen, Y.; Jiao, K.; Roberson, E.D.; Wang, Q. Noradrenergic dysfunction in Alzheimer's disease. Front Neurosci 2015, 9, 220.

García-Lorenzo, D.; Longo-Dos Santos, C.; Ewencyk, C.; Leu-Semenescu, S.; Gallea, C.; Quattrocchi, G; Pita Lobo, P.; Poupon, C.; Benali, H.; Arnulf, I; Vidailhet, M.; Lehericy, S. The Coeruleus/subcoeruleus complex in rapid eye movement sleep behavior disorders in Parkinson's disease. Neuroimage 2013, 136, 2120-2129. 
615 German, D.C.; Walker, B.S.; Manaye, K.; Smith, W.K.; Woodward, D.J.; North, A.J. The human

616 locus coeruleus: computer reconstruction of cellular distribution. J. Neurosci. 1988, 8, 1776-

6171788.

618 Graham, D.G. On the origin and significance of neuromelanin. Arch. Pathol. Lab. Med. 1979, 619 103, 359-362.

620 Grudzien, A.; Shaw, P.; Weintraub, S.; Bigio, E.; Mash, D.C.; Mesulam, M.M. Locus coeruleus

621 neurofibrillary degeneration in aging, mild cognitive impairment and early Alzheimer's disease.

622 Neurobiol Aging 2007, 28, 327-335.

623 Halliday, G.M.; Fedorow, H.; Rickert, C.H.; Gerlach, M.; Riederer, P.; Double, K.L. Evidence

624 for specific phases in the development of human neuromelanin. J Neural Transm (Vienna) 2006,

$625113,721-728$.

626 Hamilton, M. A rating scale for depression. J. Neurol. Neurosurg. Psychiatry 1960, 23, 56-62.

627 Heneka, M.T.; Ramanathan, M.; Jacobs, A.H.; Dumitrescu-Ozimek, L.; Bilkei-Gorzo, A.;

628 Debeir, T.; Sastre, M.; Galldiks, N.; Zimmer, A.; Hoehn, M.; Heiss, W-D.; Klockgether T.;

629 Staufenbiel, M. Locus Coeruleus Degeneration Promotes Alzheimer Pathogenesis in Amyloid

630 Precursor Protein 23 Transgenic Mice. J Neurosci 2006, 26, 1343-1354.

631 Hermida, A.P.; McDonald, W.M.; Steenland, K.; Levey, A. The association between late-life 632 depression, mild cognitive impairment and dementia: is inflammation the missing link? Expert

633 Rev Neurother 2012, 12, 1339-1350.

634 Isaias, I.U.; Trujillo, P.; Summers, P.; Marotta, G.; Mainardi, L.; Pezzoli, G.; Zecca, L.; Costa, 635 A. Neuromelanin Imaging and Dopaminergic Loss in Parkinson's Disease. Front Aging Neurosci 636 2016, 8, 196.

637 Jack, C.R.; Bennett, D.A.; Blennow, K.; Carrillo, M.C.; Dunn, B.; Budd Haeberlein, S.; 638 Holtzman, D.M.; Jagust, W.; Jessen, F.; Karlawish, J.; Liu, E.; Molinuevo, J.L.; Montine, T.; 639 Phelps, C.; Rankin, K.P.; Rowe, C.C.; Scheltens, P.; Siemers, E.; Snyder, H.M.; Sperling, R.; 640 Collaborators. NIA-AA Research Framework: Toward a biological definition of Alzheimer's 641 disease. Alzheimer's Dement 2018, 14, 535-562.

642 Keren, N.I.; Taheri, S.; Vazey, E.M.; Morgan, P.S.; Granholm, A.-C.E.; Aston-Jones, G.S.; 643 Eckert, M.A. Histologic validation of locus coeruleus MRI contrast in post-mortem tissue. 644 NeuroImage 2015, 113, 235-245.

645 Leonard, B.E. The role of noradrenaline in depression: a review. J. Psychopharmacol. (Oxford) 646 1997, 11, S39-47.

647 Liu, K.Y.; Marijatta, F.; Hämmerer, D.; Acosta-Cabronero, J.; Düzel, E.; Howard, R.J. Magnetic 648 resonance imaging of the human locus coeruleus: A systematic review. Neurosci Biobehav Rev $6492017,83,325-355$.

650 Liu, K.Y.; Acosta-Cabronero, J.; Cardenas-Blanco, A.; Loane, C.; Berry, A.J.; Betts, M.J.; 651 Kievit, R.A.; Henson, R.N.; Düel, E.; Cam-CAN; Howard, R.; Hämmerer, D. In vivo 
652 visualization of age-related differences in the locus coeruleus. Neurobiol Aging 2019, 74, 101653111.

654 Lobo, A., Saz, P., Marcos, G., Día, J.L., de la Cámara, C., Ventura, T., Morales Asín, F., 655 Pascual, F.L., Montañés, J.A., Aznar, S. Revalidación y normalización del miniexamen 656 cognoscitivo (primera versión en castellano del Minimental State Examination) en la población 657 general geriátrica. Med Clin (Barc) 1999, 112, 767-774.

658

659 Loughlin, S.E.; Foote, S.L.; Fallon, J.H. Locus coeruleus projections to cortex: topography, 660 morphology and collateralization. Brain Res. Bull. 1982, 9, 287-294.

661

662 Loughlin, S.E.; Foote, S.L.; Bloom, F.E. Efferent projections of nucleus locus coeruleus:

663 topographic organization of cells of origin demonstrated by three-dimensional reconstruction.

664 Neuroscience 1986, 18, 291-306.

665

666

Loughlin, S.E.; Foote, S.L.; Grzanna, R. Efferent projections of nucleus locus coeruleus:

667

668

669 Marien, M.R.; Colpaert, F.C.; Rosenquist, A.C. Noradrenergic mechanisms in neurodegenerative 670 diseases: A theory. Brain Res Rev 2004, 45, 38-78.

671

672 Matsuura, K.; Maeda, M.; Yata, K.; Ichiba, Y.; Yamaguchi, T.; Kanamaru, K.; Tomimoto, H. 673 Neuromelanin magnetic resonance imaging in Parkinson's disease and multiple system atrophy. 674 Eur. Neurol. 2013, 70, 70-77.

675

676 Miyoshi, F.; Ogawa, T.; Kitao, S. -i; Kitayama, M.; Shinohara, Y.; Takasugi, M.; Fujii, S.;

677 Kaminou, T. Evaluation of Parkinson disease and Alzheimer disease with the use of

678 neuromelanin MR imaging and (123)I-metaiodobenzylguanidine scintigraphy. AJNR Am J

679 Neuroradiol 2013, 34, 2113-2118.

680 Modrego, P.J.; Ferrandez, J. Depression in patients with mild cognitive impairment increases the 681 risk of developing dementia of Alzheimer type: a prospective cohort study. Arch Neurol 2004, $68261,1290-1293$.

683 Moret, C.; Briley, M. The importance of norepinephrine in depression. Neuropsychiatr Dis Treat 684 2011, 7, 9-13.

685 Morris, J.C. The Clinical Dementia Rating (CDR): current version and scoring rules. Neurology 686 1993, 43, 2412-2414.

687 Mukai, M.; Sugaya, K.; Yabe, I.; Goto, Y.; Yokochi, F.; Miyamoto, K.; Cai, H.; Sasaki, H.; 688 Matsubara, S. Neuromelanin MRI in a family with mitochondrial parkinsonism harboring a 689 Y955C mutation in POLG1. Parkinsonism Relat. Disord. 2013, 19, 821-824. 
690 Mulvey, B.; Bhatti, D.L.; Gyawali, S.; Lake, A. M.; Kriaucionis, S.; Ford, C.P.; Bruchas, M.R.; 691 Heintz, N.; Dougherty, J.D. Molecular and Functional Sex Differences of Noradrenergic 692 Neurons in the Mouse Locus Coeruleus. Cell Reports 2018, 23, 2225-2235.

693

694 Ohtsuka, C.; Sasaki, M.; Konno, K.; Kato, K.; Takahashi, J.; Yamashita, F.; Terayama, Y.

695 Differentiation of early-stage parkinsonisms using neuromelanin-sensitive magnetic resonance 696 imaging. Parkinsonism Relat. Disord. 2014, 20, 755-760.

697 Panza, F.; Frisardi, V.; Capurso, C.; D'Introno, A.; Colacicco, A. M.; Imbimbo, B. P.; Santamato, 698 A.; Vendemiale, G.; Seripa, D.; Pilotto, A.; Capurso, A.; Solfrizzi, V. Late-life depression, mild 699 cognitive impairment, and dementia: possible continuum?. The Am J Geriatr Psychiatry 2010, $700 \quad 18,98-116$.

701 Petersen, R.C. Mild cognitive impairment as a diagnostic entity. J. Intern. Med. 2004, 256, 183702194.

703 Petersen, R.C.; Doody, R.; Kurz, A.; Mohs, R.C.; Morris, J.C.; Rabins, P.V.; Ritchie, K.; Rossor, 704 M.; Thal, L.; Winblad, B. Current concepts in mild cognitive impairment. Arch. Neurol. 2001, $705 \quad 58,1985-1992$.

706 Priovoulos, N.; Jacobs, H.; Ivanov, D.; Uludağ, K.; Verhey, F.; Poser, B. A. High-resolution in 707 vivo imaging of human locus coeruleus by magnetization transfer MRI at $3 \mathrm{~T}$ and

708 7T. NeuroImage 2018, 168, 427-436.

709 Ramani, A.; Dalton, C.; Miller, D.H.; Tofts, P.S.; Barker, G.J. Precise estimate of fundamental 710 in-vivo MT parameters in human brain in clinically feasible times. Magn Reson Imaging 2002, $71120,721-731$.

712 Samuels, E.R.; Szabadi, E. Functional neuroanatomy of the noradrenergic locus coeruleus: its 713 roles in the regulation of arousal and autonomic function part I: principles of functional

714 organisation. Curr Neuropharmacol 2008, 6, 235-253.

715 Sasaki, M.; Shibata, E.; Ohtsuka, K.; Endoh, J.; Kudo, K.; Narumi, S.; Sakai, A. Visual

716 discrimination among patients with depression and schizophrenia and healthy individuals using

717 semiquantitative color-coded fast spin-echo T1-weighted magnetic resonance imaging.

718 Neuroradiology 2010, 52, 83-89.

719 Sasaki, M.; Shibata, E.; Tohyama, K.; Takahashi, J.; Otsuka, K.; Tsuchiya, K.; Takahashi, S.;

720 Ehara, S.; Terayama, Y.; Sakai, A. Neuromelanin magnetic resonance imaging of locus

721 coeruleus and substantia nigra in Parkinson's disease. Neuroreport 2006, 17, 1215-1218

722 Sheehan, D.V.; Lecrubier, Y.; Sheehan, K.H.; Amorim, P.; Janavs, J.; Weiller, E.; Hergueta, T.;

723 Baker, R.; Dunbar, G.C. The Mini-International Neuropsychiatric Interview (M.I.N.I.): the

724 development and validation of a structured diagnostic psychiatric interview for DSM-IV and

725 ICD-10. J Clin Psychiatry 1998, 59 Suppl 20, 22-33;quiz 34-57. 
726 Sheikh, J.I.; Yesavage, J.A. Geriatric Depression Scale (GDS): Recent evidence and

727

728

729

730

731

732

733

734

735

736

737

738

739

740

741

742

743

744

745

746

747

748

749

750

751

752

753

754

755

756

757

758

759

760

761 development of a shorter version. Clinical Gerontologist: The Journal of Aging and Mental Health 1986, 5, 165-173.

Shibata, E.; Sasaki, M.; Tohyama, K.; Kanbara, Y.; Otsuka, K.; Ehara, S.; Sakai, A. Age-related changes in locus ceruleus on neuromelanin magnetic resonance imaging at 3 Tesla. Magn Reson Med Sci 2006, 5, 197-200.

Shibata, E.; Sasaki, M.; Tohyama, K.; Otsuka, K.; Endoh, J.; Terayama, Y.; Sakai, A. Use of neuromelanin-sensitive MRI to distinguish schizophrenic and depressive patients and healthy individuals based on signal alterations in the substantia nigra and locus ceruleus. Biol. Psychiatry 2008, 64, 401-406.

Shima, T.; Sarna, T.; Swartz, H.M.; Stroppolo, A.; Gerbasi, R.; Zecca, L. Binding of iron to neuromelanin of human substantia nigra and synthetic melanin: an electron paramagnetic resonance spectroscopy study. Free Radic Biol Med 1997, 23, 110-119.

Sled, J.G.; Pike, G.B. Quantitative imaging of magnetization transfer exchange and relaxation properties in vivo using MRI. Magn Reson Med 2001, 46, 923-931.

Stahl, S.M. Stahl's essential psychopharmacology: Neuroscientific basis and practical applications, 3rd ed; Stahl's essential psychopharmacology: Neuroscientific basis and practical applications, 3rd ed; Cambridge University Press: New York, NY, US, 2008.

Steffens, D.C. Depressive symptoms and mild cognitive impairment in the elderly: an ominous combination. Biol Psychiatry 2012, 71, 762-764.

Szot, P.; White, S.S.; Greenup, J.L.; Leverenz, J.B.; Peskind, E.R.; Raskind, M.A. Compensatory changes in the noradrenergic nervous system in the locus ceruleus and hippocampus of postmortem subjects with Alzheimer's disease and dementia with Lewy bodies. J. Neurosci. 2006, 26, 467-478.

Takahashi, J.; Shibata, T.; Sasaki, M.; Kudo, M.; Yanezawa, H.; Obara, S.; Kudo, K.; Ito, K.; Yamashita, F.; Terayama, Y. Detection of changes in the locus coeruleus in patients with mild cognitive impairment and Alzheimer's disease: High-resolution fast spin-echo T1-weighted imaging. Geriatr Gerontol Int 2015, 15, 334-340.

Tosk, J.M.; Holshouser, B.A.; Aloia, R.C.; Hinshaw, D.B.; Hasso, A.N.; MacMurray, J.P.; Will, A.D.; Bozzetti, L.P. Effects of the interaction between ferric iron and L-dopa melanin on T1 and T2 relaxation times determined by magnetic resonance imaging. Magn Reson Med 1992, 26, 4045.

Trujillo, P.; Summers, P.E.; Ferrari, E.; Zucca, F.A.; Sturini, M.; Mainardi, L.T.; Cerutti, S.; Smith, A.K.; Smith, S.A.; Zecca, L.; Costa, A. Contrast mechanisms associated with neuromelanin-MRI. Magn Reson Med 2017, 78, 1790-1800.

Peer) reviewing PDF | (2020:04:48357:3:0:NEW 23 Dec 2020) 
762 Trujillo, P.; Petersen, K.J.; Cronin, M.J.; Lin, Y.C.; Kang, H.; Donahue, M.J.; Smith, S.A.; 763 Claassen, D.O. Quantitative magnetization transfer imaging of the human locus coeruleus. 764 Neuroimage 2019, 15, 191-198.

765 Watanabe, T.; Tan, Z.; Wang, X.; Martinez-Hernandez, A.; Frahm, J. Magnetic resonance 766 imaging of noradrenergic neurons. Brain Struct Funct 2019, 224, 1609-1625.

767 Wechsler, D. Escala de inteligencia de Wechsler para adultos-III; TEA Ediciones: Madrid, 768 Spain, 1999.

769 Wechsler, D. WMS-III. Escala de Memoria Wechsler - III; TEA Ediciones: Madrid, Spain, 2004.

770 Yakovlev, P.I.; Lecours, A.R. The myelogenetic cycles of regional maturation of the brain. 771 Regional Development of the Brain in Early Life A. Blackwell Scientific: Oxford, 1967.

772 Yesavage, J.A.; Brink, T.L.; Rose, T.L.; Lum, O.; Huang, V.; Adey, M.; Leirer, V.O.

773 Development and validation of a geriatric depression screening scale: a preliminary report. $J$

774 Psychiatr Res 1982, 17, 37-49.

775 Zarow, C.; Lyness, S.A.; Mortimer, J.A.; Chui, H.C. Neuronal loss is greater in the locus 776 coeruleus than nucleus basalis and substantia nigra in Alzheimer and Parkinson diseases. Arch 777 Neurol 2003, 60, 337-341. 


\section{Table $\mathbf{1}$ (on next page)}

Characteristics of the study sample.

Abbreviations: aMCl, amnestic Mild Cognitive Impairment; df, degrees of freedom; GDS, Geriatric Depression Scale; HDRS, Hamilton Depression Rating Scale; MDD, late-life Major Depression Disorder; MMSE, Mini-

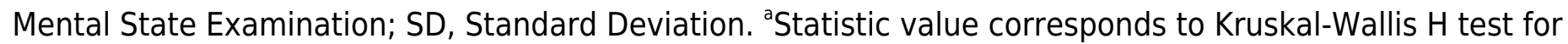
continuous variables and Chi-Square test for categorical variables.

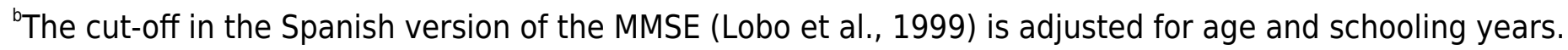
Scores higher than 23 for literate people and higher than 18 for illiterates indicate preserved general cognitive functioning.

${ }^{\mathrm{c}}$ Evaluated with the delayed recall test from the Wechsler Memory Scale III (WMS-III). 
1 Table 1. Characteristics of the study sample.

2

\begin{tabular}{|c|c|c|c|c|}
\hline & $\begin{array}{c}\text { MDD patients } \\
\qquad \mathbf{n}=\mathbf{3 7}\end{array}$ & $\begin{array}{c}\text { aMCI patients } \\
n=21\end{array}$ & $\begin{array}{l}\text { Controls } \\
\qquad \mathbf{n}=\mathbf{3 1}\end{array}$ & $\begin{array}{c}\text { Statistic } \\
\text { (p value) }^{\mathrm{a}} \\
\text { df }\end{array}$ \\
\hline \multicolumn{5}{|c|}{ Sociodemographic characteristics } \\
\hline $\begin{array}{l}\text { Age, years; mean } \\
\text { (SD) [range] }\end{array}$ & $68(4.1)[60-74]$ & $\begin{array}{c}71.5(2.7) \quad[67- \\
76]\end{array}$ & $\begin{array}{c}67.7(4.1) \\
{[60-75]}\end{array}$ & $\begin{array}{c}H=1.38 \\
(0.001) \\
2\end{array}$ \\
\hline Sex, male; n (\%) & $11(29.7)$ & $9(42.9)$ & $11(35.5)$ & $\begin{array}{c}X^{2}=1.03 \\
(0.599) \\
2\end{array}$ \\
\hline \multicolumn{5}{|c|}{ Clinical characteristics } \\
\hline $\begin{array}{c}\text { HDRS; mean }(\mathrm{SD}) \\
\text { [range }]\end{array}$ & $12.1(7.1)[1-28]$ & $3.8(3)[0-8]$ & $\begin{array}{c}1.3(2.8) \\
{[0-15]}\end{array}$ & $\begin{array}{c}\mathrm{H}=50.85 \\
(<0.0001) \\
2\end{array}$ \\
\hline $\begin{array}{c}\text { GDS; mean (SD) } \\
\text { [range] }\end{array}$ & $5.6(4.3)[0-13]$ & $2.3(2.2)[0-9]$ & $\begin{array}{c}1.23(2.3) \\
{[0-12]}\end{array}$ & $\begin{array}{c}\mathrm{H}=27.7 \\
(<0.0001) \\
2\end{array}$ \\
\hline $\begin{array}{c}\text { MMSE; mean (SD) } \\
{[\text { range }]^{\mathrm{b}}}\end{array}$ & $\begin{array}{c}25.9(3.3) \\
{[13-30]}\end{array}$ & $\begin{array}{c}25.8(2.6) \\
{[20-29]}\end{array}$ & $\begin{array}{c}28.9(1.49) \\
{[24-30]}\end{array}$ & $\begin{array}{c}H=32.68 \\
(<0.0001) \\
2\end{array}$ \\
\hline $\begin{array}{l}\text { Long Term Memory } \\
\text { c; (SD) [range] }\end{array}$ & $\begin{array}{c}5.08(2.54) \\
{[0-10]}\end{array}$ & $\begin{array}{c}1.95(1.28) \\
{[0-4]}\end{array}$ & $\begin{array}{c}8.32(1.72) \\
{[6-12]}\end{array}$ & $\begin{array}{l}\mathrm{H}=53.93 \\
(<0.0001)\end{array}$ \\
\hline
\end{tabular}


4 Abbreviations: aMCI, amnestic Mild Cognitive Impairment; df, degrees of freedom; GDS, 5 Geriatric Depression Scale; HDRS, Hamilton Depression Rating Scale; MDD, late-life Major 6 Depression Disorder; MMSE, Mini-Mental State Examination; SD, Standard Deviation.

7 a Statistic value corresponds to Kruskal-Wallis $\mathrm{H}$ test for continuous variables and Chi-Square

8 test for categorical variables.

$9 \mathrm{~b}$ The cut-off in the Spanish version of the MMSE (Lobo et al., 1999) is adjusted for age and schooling 10 years. Scores higher than 23 for literate people and higher than 18 for illiterates indicate preserved general 11 cognitive functioning.

$12{ }^{\mathrm{c}}$ Evaluated with the delayed recall test from the Wechsler Memory Scale III (WMS-III). 


\section{Table 2 (on next page)}

Antidepressant treatment at the time of the study

Abbreviations: aMCl, amnestic Mild Cognitive Impairment; MDD, late-life Major Depression Disorder; SD, Standard Deviation. 
1 Table 2. Antidepressant treatment at the time of the study.

2

n (\%) [dose-range in $\mathrm{mg}]$

MDD patients $\mathbf{n}=\mathbf{3 7}$

aMCI patients $n=21$

Tricyclics

\begin{tabular}{|c|c|c|}
\hline Clomipramine & $2(5.4)[37.5-75]$ & $0(0)[0]$ \\
\hline Imipramine & $2(5.4)[75-175]$ & $0(0)[0]$ \\
\hline Amitriptyline & $1(2.7)[25]$ & $2(9.5)[20-25]$ \\
\hline
\end{tabular}

Selective serotonin reuptake inhibitors (SSRIs)

\begin{tabular}{|c|c|c|}
\hline Sertraline & $1(2.7)[200]$ & $0(0)[0]$ \\
\hline Citalopram & $2(5.4)[10]$ & $4(19.1)[10-20]$ \\
\hline Paroxetine & $3(8.1)[20-30]$ & $0(0)[0]$ \\
\hline
\end{tabular}

Serotonin-norepinephrine reuptake inhibitor (SNRIs)

\begin{tabular}{|c|c|c|}
\hline Venlafaxine & $12(32.4)[75-300]$ & $0(0)[0]$ \\
\hline Duloxetine & $11(29.7)[60-120]$ & $0(0)[0]$ \\
\hline Desvenlafaxine & $2(5.4)[50-100]$ & $0(0)[0]$ \\
\hline
\end{tabular}

Other antidepressants

\begin{tabular}{|c|c|c|}
\hline Mirtazapine & $7(18.9)[15-30]$ & $2(9.5)[7.5-15]$ \\
\hline Bupropion & $5(13.5)[150-300]$ & $0(0)[0]$ \\
\hline Vortioxetine & $1(2.7)[10]$ & $0(0)[0]$ \\
\hline Agomelatine & $2(5.4)[25]$ & $0(0)[0]$ \\
\hline Trazodone & $4(10.8)[50-100]$ & $7(33.3)[50-100]$ \\
\hline
\end{tabular}

3

Abbreviations: aMCI, amnestic Mild Cognitive Impairment; MDD, late-life Major Depression Disorder; SD, Standard Deviation. 


\section{Table 3(on next page)}

Average LC Contrast Ratios.

Abbreviations: aMCl, amnestic Mild Cognitive Impairment; HCs, Healthy Controls; LC: Locus

Coeruleus; MDD, late-life Major Depressive Disorder; SD, Standard Deviation; SNRIs, Serotonin and Norepinephrine reuptake inhibitors. 
1 Table 3. Average LC Contrast Ratios.

\begin{tabular}{|c|c|c|}
\hline & $\begin{array}{l}\text { Average LC Contrast } \\
\text { Ratio } \\
\text { Intensity: mean (SD) }\end{array}$ & $\begin{array}{c}\text { Standardized residual of } \\
\text { Average LC Contrast Ratio } \\
\text { (sraLCCR) } \\
\text { Intensity: mean (SD) }\end{array}$ \\
\hline MDD whole group $(n=37)$ & $0.194(0.059)$ & $-0.450(0.0728)$ \\
\hline MDD with aMCI (n=9) & $0.195(0.084$ & $-0.474(1.016)$ \\
\hline MDD without aMCI $(\mathrm{n}=28)$ & $0.194(0.052)$ & $-0.442(0.633)$ \\
\hline $\operatorname{aMCI}(\mathrm{n}=21)$ & $0.234(0.079)$ & $0.257(0.946)$ \\
\hline $\operatorname{HCs}(n=31)$ & $0.260(0.093)$ & $0.363(1.096)$ \\
\hline \multicolumn{3}{|c|}{ MDD split according to SNRIs treatment } \\
\hline MDD taking SNRIs $(n=25)$ & $0.180(0.045)$ & $-0.621(0.538)$ \\
\hline $\begin{array}{l}\text { MDD not taking SNRIs } \\
\qquad(\mathrm{n}=12)\end{array}$ & $0.224(0.076)$ & $-0.094(0.947)$ \\
\hline
\end{tabular}

2

3 Abbreviations: aMCI, amnestic Mild Cognitive Impairment; HCs, Healthy Controls; LC: Locus 4 Coeruleus; MDD, late-life Major Depressive Disorder; SD, Standard Deviation; SNRIs, 5 Serotonin and Norepinephrine reuptake inhibitors. 


\section{Figure 1}

Visualization and localization of the LC and the reference region

Figure 1. a): Axial, coronal and sagittal views of the dorsal pons from the modified fast spinecho T1 sequence of a representative participant (see text for details). The LC can be identified in each hemisphere as an hyperintense structure (red arrows). b): Axial, coronal and sagittal views of the dorsal pons from the high-dimensional whole-brain T1 sequence of the same participant, depicting, in red, the 10 most intense LC voxels as identified by the growing algorithm. The denormalized ROI of the LC and reference regions are depicted as red (encompassing the 10 most intense LC voxels) and blue contours, respectively.

a)

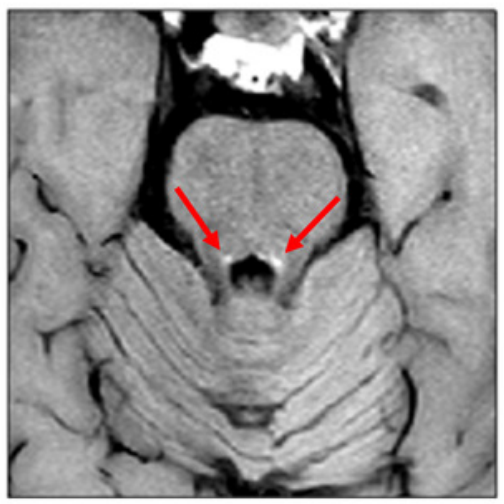

b)

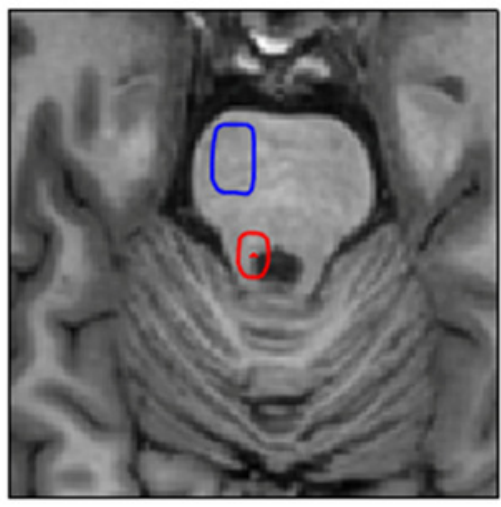

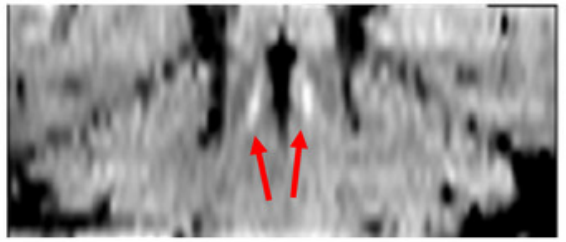
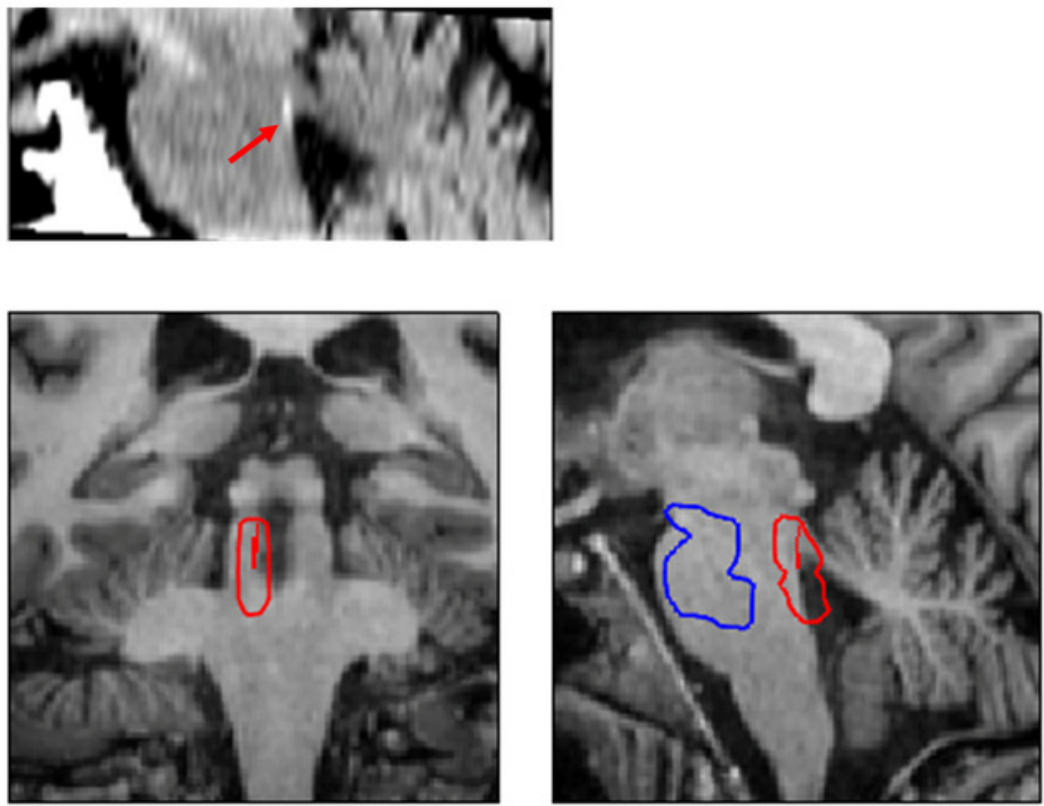


\section{Figure 2}

Boxplots depicting the average LC Contrast Ratios

Figure 2. Boxplots showing the average LCCR (age and sex adjusted, sraLCCR) for the different study groups. The individual values (dots) are overlaid for reference. a) Boxplots of the average LCCR for the three main study groups; b) Boxplots of the average LCCR splitting patients with late-life MDD as a function of SNRIs treatment. aMCl, amnestic type Mild Cognitive Impairment; HCs, Healthy Controls; LCCR, Locus Coeruleus Contrast Ratio; MDD, late-life Major Depressive Disorder. ${ }^{* *} p \leq 0.005,{ }^{* * *} p \leq 0.001$. All results remained significant after excluding outlier values. 
a)

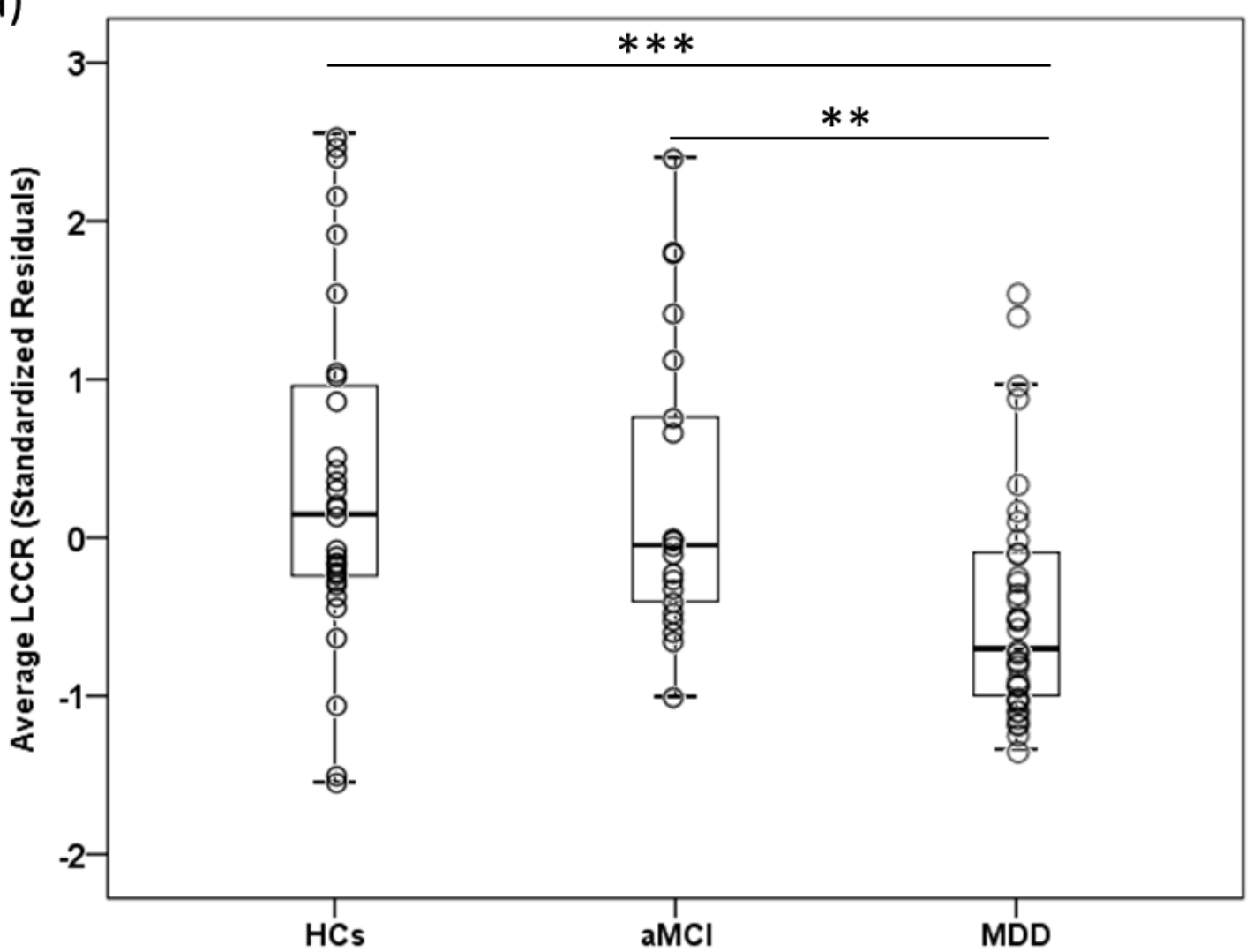

b)

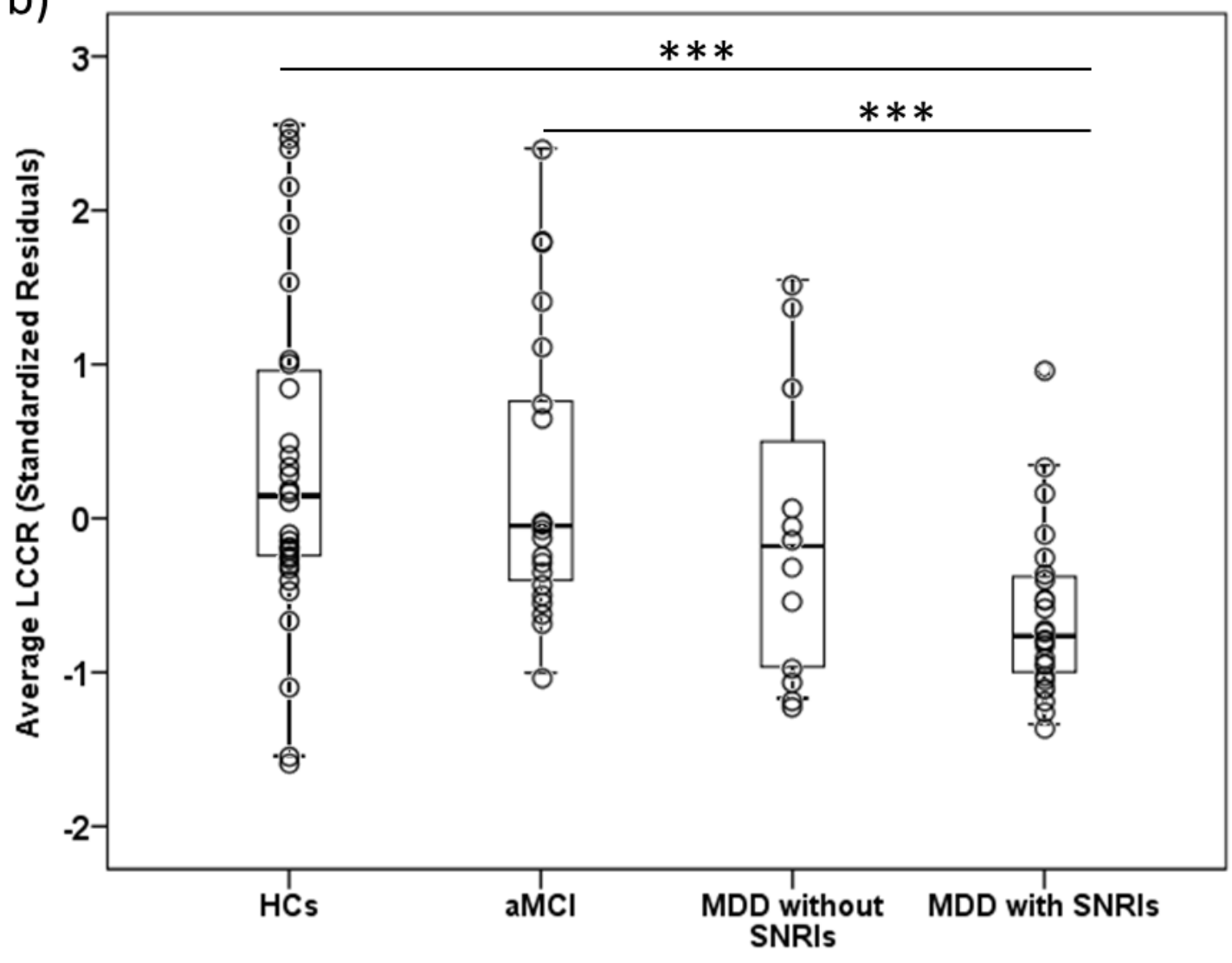

